\title{
Neue Geräte und Chemikalien
}

\section{Messung des freien Sauersloffs}

Die Fa. Electro-Nite N.V., B-3530 Houthalen, bietet einen neuentwickelten Meßkopf zum Messen des freien Sauerstoffgehaltes in Stahlschmelzen an. Der Meßkopf kann nur einmal verwendet werden. Das System, „Celox" genannt, legt zugleich Temperaturwert und freien Sauerstoffgehalt fest.

\section{Computergesteuertes Speltralphotometer}

Datacolor AG, Dietikon bei Zürich, hat ein computergesteuertes Spektralphotometer entwickelt, das speziell für den industriellen Einsatz in allen farbgebenden Industrien gedach $t$ ist. Dieses neue Farbmeßgerät wird in hohem Maße den Forderungen gerecht, die die praktische Anwendung bezüglich einfacher Handhabung, Genauigkeit, Schnelligkeit und Vielseitigkeit stellt. Dank der Ausbaubarkeit des integrierten Computersystems besteht die Möglichkeit einer individuellen betrieblichen Anpassung hinsichtlich Datenverarbeitung und Datenübermittlung. Das Gerät kann bis zum selbständigen, festprogrammierten Farbrezeptrechner erweitert werden.

\section{Durchflußmesser für Gase und Fllüssigkeiten}

Die Fa. Fischer \& Porter GmbH, Göttingen, empfiehlt einen DralldurchfuBmesser (DDM). Er arbeitet nach dem Prinzip der Schwingungsmessung in strömenden Medien. Mit strömungsmechanischen Mitteln wird ein digitales Ausgangssignal erzeugt, das dem Volumenstrom direkt proportional ist. Ein DDM besitzt keine beweglichen Teile. Seine Eichkonstante ist in weiten Grenzen von Druck, Temperatur und Dichte des strömenden Mediums unabhängig. Der Meßbereich eines DDM mit den Garantiefehlergrenzen von $\pm 1 \%$ vom Meßwert and einer Meßunsicherheit von $\pm 0,25 \%$ vom Meßwert beträgt im Mittel 1:10 bis 1:20. Der DDM kann in beliebiger Lage in eine Rohrleitung eingebaut werden.

\section{Quadrupol-Massenspektrometer für die Analytik}

Die Finnigan GmbH, München, hat ein Quadrupol-Massenspektrometer in Kombination mit einem Gas-Chromatographen und einem Fest.örperdirekteinlaB angeboten. Durch Koppelung mit elektronischer Datenverarbeitung liegen die Meßergebnisse in kürzester Zeit vor.

\section{Zerstörungsfreie Schichtdickenmessung}

Die Helmut Fischer GmbH \& Co, Maichingen, bietet als Neuentwicklung das ,Anotest"-Meßgerät an. Es dient zur Qualitätsprüfung der Nachverdichtung von anodischen Oxidschichten für Aluminium durch Bestimmung des Scheinleitwertes und Verlustfaktors.

\section{Meßgeräte für die Wasseraufbereitung}

Die Analytical Meßinstrumente GmbH \& Co KG, Düsseldorf, bietet eine komplette Reihe von elektronischen Meßgeräten zur automatischen Regelung und Messung in Wasseraufbereitungsanlagen, speziell auch für Abwasseranlagen, an. Im einzelnen gehören hierzu: Leitfähigkeitsmeßgeräte, $\mathrm{pH}$ Meter, Cyan- und Chrom-BestimmungsmeBgeräte. Neu sind eine anzeigende und zählende Strömungsmengenmeßeinrichtung, Trübungsmeßgeräte sowie Meßsonden und Zubehör für solche Anlagen.

\section{Zubehör für schreibende Meßgeräte}

Die Dia-Nielsen GmbH, Düren, bietet eine umfangreiche Palette von schreibendem Zubehör für Meßgeräte an. Rund 4000 Artikel dienen dazu, einwandfreie Diagramm-Aufzeichnungen zu erzielen. Hervorzuheben sind die Dia-Wegwerf-Faserschreibfedern, die in Verbindung mit Dia-Registriertinten jederzeit ein sofortiges Anschreiben sichern sollen. Verbraucher dürften auch an den auswechselbaren DiaFasersehreibspitzen interessiert sein, die Vorteile gegenüber herkömmlichen Metallcapillaren bieten. Zum Herstellungsprogramm zählen außerdem: Farbbänder, Farbfilze, Farbstäbe, Schreibelektroden sowie patentierte TEP-Schreibeinrichtungen, die in Kombination mit Tintenpatronen nur geringe Wartung erfordern.

\section{Gläserne Laborpumpe}

Eine neuartige Labor-Glaspumpe liefert die Fa. O. Fritz, Hofheim. Das Gerät besteht aus dem Pumpengehäuse, dem Pumpenïüuer (einem halbkugelförmigen, 4 fach polarisierten Magneten, der zur Vermeidung von Korrosion in Teflon ${ }^{\circledR}$ eingebettet ist) und dem äußeren, geführten Gegenmagneten (Halbhohlkugel). Die nach außen ragende Welle kann mit einem Labor-Rührmotor verbunden werden. Der teflonisierte Pumpenläufer wird nicht nur magnetisch mitgenommen, sondern ist auch magnetisch gelagert. Er läuft auf einem Glaszapfen. Mit der Pumpe können Flüssigkeiten geringer Viscosität auch dann gefördert werden, wenn sie kleine Mengen fester Stoffe enthalten.

\section{Neue Schwingmühle für die IR-Speltroskopie}

Eine neue Schwingmühle von der Bodenseewerk PerkinElmer \& Co GmbH, U̇berlingen, dient zur Vorbereitung von KBr-PreBlingen für die Infrarot-Spektroskopie. Es lassen sich feste, harte und pulverförmige Stoffe sehr fein mahlen und mischen. Zum Mahlen nicht-spröder Stoffe, z. B. von Polymeren, läßt sich die Mühle mit flüssigem Stickstoff tiefkühlen. - Das Mahlgut wird in einer Kapsel mit einer Kugel, die lose in der Kapsel liegt, durch schnelle Vibration der Kapsel zerkleinert und gemischt. Die Mahlkapseln und die Mahlkugeln bestehen aus Achat oder aus gehärtetem Chromstahl. Die Mahldauer ist zwischen 0 und 30 sec einstellbar.

\section{Klebeverbindungen}

Die Chemischen Werke Werner \& Mertz GmbH, MainzMombach, bieten den Ardal-Schmelzkleber an. Er wird in fester Form geliefert und mit einem besonderen Auftragsgerät in heißem, flüssigen Zustand aus einer Düse oder mit einer Walze aufgetragen. Das Gegenstück wird sofort aufgelegt und ist in wenigen Sekunden fest verbunden. Die Endfestigkeit ist bereits nahezu erreicht, sobald die Klebefuge abgekühlt ist. Die Schmelzkleber enthalten weder Lösungsmittel noch Weichmacher noch korrosionsfördernde Bestandteile. Sie sollen eine ausgezeichnete Adhäsion zu fast allen Materialien haben, einschließlich Polyäthylen, Polypropylen und Acetalharze. Die Scherfestigkeit erreicht bis $50 \mathrm{kp} / \mathrm{cm}^{2}$. 